\title{
A Local Variance-Based Bilateral Filtering for Artifact-Free Detail- and Edge-Preserving Smoothing
}

\author{
Cuong Cao Pham, Synh Viet Uyen Ha, and Jae Wook Jeon* \\ School of Information and Communication Engineering, Sungkyunkwan University, \\ Suwon, South Korea \\ cuongpc@skku.edu, synhha@ece.skku.edu, jwjeon@yurim.skku.ac.kr \\ http://micro.skku.ac.kr
}

\begin{abstract}
Edge-preserving smoothing has recently emerged as a crucial technique for a variety of computer vision and image processing applications. The idea is to smooth the small scale variations, while preserving edges and fine details in the image, without causing halo artifacts in detail enhancement. In this paper, we propose a modified bilateral filter model that has better behavior near edges and details than the standard model does. The edge-stopping function takes into account the intensity difference and the local variance of the filter windows, which provides insightful information about the local pixel distribution. We demonstrate the existing detail-related bilateral-based applications can achieve better results by simply switching from the standard model to our proposed model. In particular, we applied our method to detail-preserving image denoising and detail enhancement.
\end{abstract}

Keywords: Edge-preserving smoothing, bilateral filter, detail enhancement, image denoising.

\section{Introduction}

Edge-preserving smoothing plays a crucial role in a variety of applications in computer vision and image processing. The filtering progress will smooth the noise, while preserving edges and fine details of the image. Both the smoothing result or base layer and residual detail layer can be used in different ways, depending on each application. For instance, the details can be boosted in applications, such as detail enhancement, while in image de-noising they will be discarded.

\footnotetext{
* This research was supported by the MKE (The Ministry of Knowledge Economy), Korea, under the ITRC (Information Technology Research Center) support program supervised by the NIPA (National IT Industry Promotion Agency) (NIPA2011-(C1090-1121-0008)), and by Priority Research Centers Program through the National Research Foundation of Korea (NRF) funded by the Ministry of Education, Science and Technology (2011-0018397).
} 
State of the art techniques that enable this kind of filter include anisotropic diffusion (AD) [19], bilateral filter (BLF) 23, weighted least squares filter (WLS) 12 and guided image filter (GIF) [14. Each has its advantages and disadvantages, so we need to consider the balance between the quality of the results and the computational cost. By taking this consideration, GIF and BLF would be the most suitable choices. Especially, BLF has become the de facto standard for computer vision and image processing applications due to its simplicity, flexibility and appealing characteristics [18].

The main challenges of BLF are its time consumption and production of reversed gradient artifacts near edges in detail enhancement technique. While the time taken can be reduced using fast approximated techniques [7/10 17/20], causing reversed gradient artifacts in detail enhancement and filtering meaningful fine details in image de-noising are unresolved issues.

In this paper, we propose a modified BLF model that has better behavior near edges than the standard model does. We realize that the halo artifacts, that occur in detail enhancement, come from the weak discrimination between pixel pairs in the local window. In general, the simple intensity difference of the standard model is insufficient to determine which pixels should be smoothed and which detail- and edge-pixels should be preserved. The proposed method takes into account the intensity difference as does the standard model, and the local variances of the image, which provide insightful information about local pixel distribution. Experiments show bilateral-based detail enhancement and detailpreserving image denoising can achieve better results by simply switching from the existing standard model to our improved model.

The remainder of this paper is organized as follows. Section 2 discussed various existing edge-preserving smoothing techniques. Section 3 presents the proposed method with the use of local variance. Section 4 presents experimental results to compare our method to methods from the literature. Finally, this paper is drawn to a conclusion and future work outlined in Section 5.

\section{Related Work}

Several state of the art edge-preserving smoothing techniques have been developed and applied in a variety of computer vision and image processing applications in the last decade. The ideal edge-preserving filter must smooth the small scale variations or noise of the image and preserve edges and fine details without causing any halo artifacts near edges in detail enhancement. In particular, it must neither blur nor sharpen the edges.

Such a filter was first used for scale-space image segmentation and edge detection known as anisotropic diffusion (AD) [19]. Generally, AD strongly smooths the low gradient regions, while preventing averaging across the strong edges where the gradient magnitude is large. This is the reason why AD can smooth noise while preserving edge structures. However, it has three major drawbacks. First, the meaningful fine details of an image are unexpectedly removed due to its over-smooth characteristic. Second, the edges are over-sharpened in the fi- 
nal cumulative result. Finally, the non-linear iterative process of $\mathrm{AD}$ is slow to converge.

Although there are some improved anisotropic diffusion models, such as robust anisotropic diffusion [3], variance-based [6], LCIS [24], these limitations cannot be overcome thoroughly. BLF is increasingly widely used due to its appealing characteristics. It was original developed in 1121], and then popularized in [23] with the name bilateral filter. It is a non-iterative, non-linear weighted averaging filter that smooths noise, whilst preserving edges. A weighted mean of neighbor pixels is calculated to produce each output pixel in the filtered result. The weight is computed by the product of the spatial and intensity range domain. Let $I_{p}$ be the intensity value at pixel $p$; then, BLF is defined by:

$$
B L F(I)_{p}=\frac{\sum_{q} g_{\sigma_{s}}(p, q) f_{\sigma_{r}}\left(I_{p}, I_{q}\right) I_{q}}{\sum_{q} g_{\sigma_{s}}(p, q) f_{\sigma_{r}}\left(I_{p}, I_{q}\right)}
$$

where kernel functions $g_{\sigma_{s}}$ and $f_{\sigma_{r}}$ are spatial and range domain, respectively. Parameters $\sigma_{s}$ and $\sigma_{r}$ determine the weights in each domain, represented by a Gaussian function:

$$
\begin{gathered}
g_{\sigma_{s}}(p, q)=\exp \left(-\|p-q\|^{2} / 2 \sigma_{s}^{2}\right) \\
f_{\sigma_{r}}\left(I_{p}, I_{q}\right)=\exp \left(-\left|I_{p}-I_{q}\right|^{2} / 2 \sigma_{r}^{2}\right)
\end{gathered}
$$

BLF can perform the smoothing process in a single iteration and overcome the over-sharpen edges characteristic of anisotropic diffusion. The weights assigned to neighbor pixels in spatial domain depend on their locations, while intensity values determine the weights in range domain. Specifically, the spatial domain reduces the weight of distant pixels, and the range domain reduces the weight of pixels that are different from the center pixel in terms of intensity value. The degree of smoothing is controlled by adjusting the value of $\sigma_{r}$. BLF becomes equivalent to the Gaussian low-pass filter when $\sigma_{r}$ increases. Hence, this value must be sufficiently small to avoid filtering fine features.

The main disadvantages of BLF are its time consumption and production of gradient reversal artifacts near edges in detail enhancement. Several fast approximated techniques have been investigated to reduce the time-taken [7/0 17/20]. The main concepts of these acceleration schemes can be found in 18. The halo artifacts that occur in detail enhancement can be controlled using a postprocessing algorithm proposed in [15. Our improved model greatly reduces these artifacts, without using such a post-processing step. Moreover, the detailpreserving characteristic of our model is another advantage compared to the standard model.

Some improvements of BLF that applied adaptive parameters instead of fixed parameters have been developed. Wong [26] included the local phase coherence into both spatial and range domain of BLF to improve the noise reducing and detail-preserving ability. However, the gradient reversal artifacts and detail enhancement have not been evaluated and discussed. While the adaptive BLF 
developed in [27] tends to enhance the sharpness of the noisy blurred image, not to boost the detail as our stated problem.

To address the second problem of BLF, Farbman et al. [12 also introduced the weighted least squares (WLS) filter that better preserves the edges than BLF does and is able to perform halo-free image decomposition of an input image. It can produce a high quality smoothed and enhanced result, without suffering obvious artifacts, but requires solving a large sparse linear system. This leads us to another challenge.

Subr et al. 22] favored using the local extrema-based filter (LEF) instead of WLS filter. The output image is computed by averaging $\mathrm{min} / \mathrm{max}$ envelopes, which are interpolated from $\mathrm{min} / \mathrm{max}$ extrema using the edge-preserving interpolation scheme [16. However, this method tends to remove details even in high contrast textured regions. Furthermore, it causes incomplete smoothing of the oscillation in a single iteration, and requires solving $k$ number of large sparse linear system $(k \geq 2)$.

In a notable recent work, He et al. [14] made use of the local linear transform model to propose guided image filtering (GIF). Although GIF can reduce the gradient reversal artifacts of BLF, and can be implemented using a fast algorithm, the details are not well preserved, and the contrast of its resultant output is reduced, as in the case of BLF.

In summary, we need to trade off the result quality and computational cost. Among existing methods, the bilateral-based filter is still widely used and would have become the de facto standard for computer vision applications [18]. The next section presents our improved BLF model with the use of local variance. Experiments show our method can achieve better results than conventional BLF in detail enhancement and image de-noising.

\section{Proposed Bilateral Filter Model}

In this section, we present a modified BLF model that better preserves edges and fine details compared to the standard model. The edge-stopping function will take into account the intensity difference and the local variance of the filter windows. First, we briefly analyze the variance information of the local windows, and then apply it to the proposed model. The related analysis can be found in [6] for degraded background images, while our analysis strongly focuses on the windows nears edges.

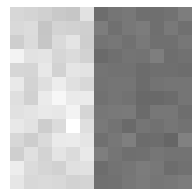

(a)

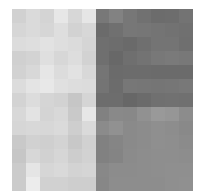

(b)

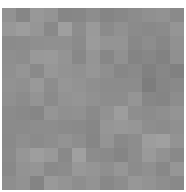

(c)

Fig. 1. Local windows of size $13 \times 13$ of an input image 
Table 1. Comparison of our method and existing edge-preserving smoothing techniques

\begin{tabular}{|c|c|c|c|}
\hline Filtering & Smoothed & Preserved & Assumption \\
\hline AD & Low gradient region & Large gradient region & $\begin{array}{c}\text { Gradient magnitude at } \\
\text { edges is large }\end{array}$ \\
\hline BLF & Low gradient region & Large intensity difference & Noise is low contrast \\
\hline GIF & Low variance region & High variance region & Noise is low contrast \\
\hline WLS & Low gradient region & Large gradient region & $\begin{array}{c}\text { Gradient magnitude at } \\
\text { edges is large }\end{array}$ \\
\hline LEF & $\begin{array}{c}\text { Oscillations between min } \\
\text { and max local extrema }\end{array}$ & $\begin{array}{c}\text { High variance of } \\
\text { neighboring local extrema }\end{array}$ & $\begin{array}{c}\text { Detail can be low } \\
\text { or high contrast }\end{array}$ \\
\hline Our & $\begin{array}{c}\text { Low gradient, } \\
\text { low variance region }\end{array}$ & $\begin{array}{c}\text { Large intensity difference, } \\
\text { high variance region }\end{array}$ & Noise is low contrast \\
\hline
\end{tabular}

\subsection{Local Variance Analysis}

For the enlarged windows of an input image as shown in Fig. 1, Fig. 1(a) and 1(b) contains some step edges, while Fig. 1(c) only contains small scale oscillations. The objective of edge-preserving smoothing for such these images is to smooth the small scale oscillations and prevent their inter-region edges leaking together. The variances of these windows are $11.14,8.44$ and 0.18 , respectively. It has long been known that the local variance is calculated by:

$$
\sigma_{p}^{2}=\frac{1}{|w|} \sum_{q \in w_{p}}\left(I_{q}-\mu_{p}\right)^{2}
$$

where $\mu_{p}$ is the mean of $I$ in the local window $w_{p}$, and $|w|$ is the number of pixels in $w_{p}$. As discussed in [6], it should be normalized to make the range of variance to be compatible with the range of gradient, as in equation (5):

$$
\sigma_{p, N}^{2}=1+\frac{\sigma_{p}^{2}-\min \sigma_{p}^{2}}{\max \sigma_{p}^{2}-\min \sigma_{p}^{2}} \cdot 254
$$

where $\min \sigma_{p}^{2}$ and $\max \sigma_{p}^{2}$ are the minimum and maximum local variance of the entire image. The normalized variances of these above windows are 234.79, 178.13 and 4.61, respectively.

We can observe that the variances of the local windows that contain interregion edges are larger than that in homogeneous regions. In order to preserve detail- or edge-pixels that located near the center of these windows, their neighbor pixels should have small weight in the filtering process. For instance, while computing the output of center pixels in Fig. 1(a) and 1(b) the small weights should be assigned to their neighbors. Meanwhile, a low-pass filter is applicable to homogeneous region, as Fig. 1(c) 


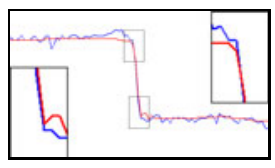

(a) WLS

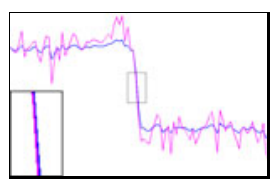

(e) WLS

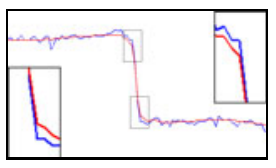

(b) GIF

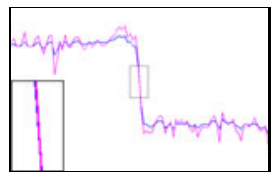

(f) GIF

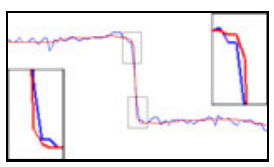

(c) BLF

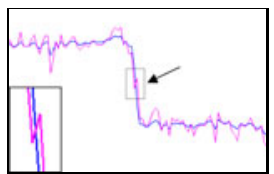

(g) BLF

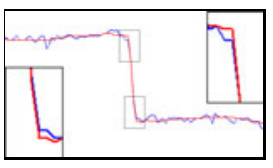

(d) Our

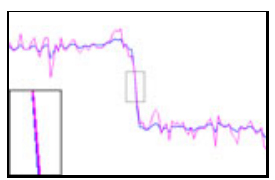

(h) Our

Fig. 2. Filter outputs (top row) and detail enhancement (bottom row) in 1-D of our method and existing methods. Parameters are configured as followed: WLS $(\lambda=0.5$, $\alpha=1.2), \operatorname{GIF}\left(r=6, \epsilon=0.05^{2}\right), \operatorname{BLF}\left(\sigma_{s}=6, \sigma_{r}=0.1\right)$ and $\operatorname{Our}\left(\sigma_{s}=6, \sigma_{v r}=0.01\right)$

\subsection{Proposed BLF Model}

Capture the notion of local variance and its insightful information about the local pixel distribution, we proposed the modified BLF model that takes into account both intensity difference and local variance. We tend to reduce the weights of local neighbors in the smoothing process of high-variance detail- or edge-pixels. The proposed BLF model with the use of local variance is given as follows:

$$
V B F(I)_{p}=\frac{\sum_{q} g_{\sigma_{s}}(p, q) f_{\sigma_{v r}}^{\prime}\left(I_{p}, I_{q}, \sigma_{p, N}^{2}\right) I_{q}}{\sum_{q} g_{\sigma_{s}}(p, q) f_{\sigma_{v r}}^{\prime}\left(I_{p}, I_{q}, \sigma_{p, N}^{2}\right)}
$$

where $g_{\sigma_{s}}$ is spatial domain defined in (2), and $f_{\sigma_{v r}}^{\prime}$ is our modified edge-stopping function,

$$
f_{\sigma_{v r}}^{\prime}\left(I_{p}, I_{q}, \sigma_{p, N}^{2}\right)=\exp \left(-\frac{1}{2}\left(\frac{\left|I_{p}-I_{q}\right| \cdot \sigma_{p, N}^{2}}{\sigma_{v r}}\right)^{2}\right)
$$

where $\sigma_{v r}$ plays the role of the smoothing parameter, the larger the value of $\sigma_{v r}$ is, the smoother the filter image will be. However, it must be much smaller than the standard deviation $\sigma_{r}$ used in the standard model in the normalized $[0 ; 1]$ intensity range value. With this proposed edge-stopping function, the small weights will be assigned to the neighbors of high-variance detail- and edge-pixels, this effect prevents two inter-region edges leaking together as the ideal filter. Meanwhile, the variances are close to one in the homogeneous regions; the proposed model becomes equivalent to the standard BLF, and noise and small scale oscillations will be smoothed. Table 1 shows the overview characteristics of our method in the context of existing techniques.

\subsection{Edge-Preserving Characteristic}

Fig. 2 visually compare our method and existing methods. Given an 1-D input signal (blue) plotted from a scan-line of an image, the filter outputs (red) are 


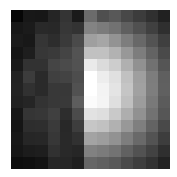

(a)

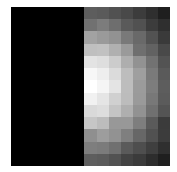

(d)

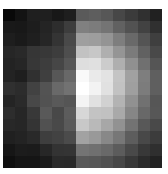

(b)

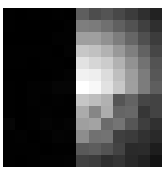

(e)

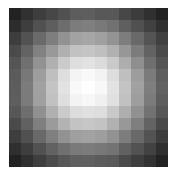

(c)

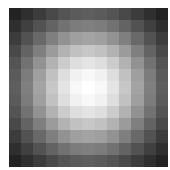

(f)

Fig. 3. Filter kernels of bilateral filter (top row) and our method (bottom row) corresponding to Fig. 1(a), 1(b), and 1(c)

shown in the top row, and the enhanced outputs (magenta) are shown in the bottom row. We can easily see that the reversed gradient artifacts only in the enhanced output of BLF (Fig. 2(g), , because the edge-pixel and its neighbors have leaked together in the smoothed result (base layer) (Fig. 2(c)). In contrast, our method with the participation of local variance information does not suffer such artifacts, as shown in Fig. 2(h). The WLS filter and GIF yield high quality in both smoothed (Fig. 2(a) and 2(b) and enhanced output (Fig. 2(e) and 2(f)], as they have been proved previously [1214.

Fig. 3 shows the filter kernel visualization of BLF and our method corresponding to the enlarged windows of Fig. 1 Fig. 3(a) and 3(b) show the weights assigned to the pixels on the non-same region to the center pixel are still large, so the edges will not be consistently preserved for BLF. Conversely, the weights assigned to these pixels in our method are nearly zero (black), as shown in Fig. $3(\mathrm{~d})$ and $3(\mathrm{e})$. This better preserves the edge-pixels than the standard BLF does. Both filters have equivalent behavior for the low-gradient, low-variance regions, the neighbors are averaged together. As we can see in the right-most column, both filter kernels are comparable to a low-pass filter.

\subsection{Implementation}

The proposed model naturally works well with a gray-scale image. In order to process with color image, both intensity difference and local variance are calculated by Euclidean distance in CIELab color space. We can achieve better results by processing in CIELab space, rather than RGB, because the channel-wise CIELab space is more uniform than RGB $[23$ is. The algorithm is directly implemented using CUDA in this work to achieve the speed-up. The GPU implementation takes about $90 \mathrm{~ms}$ to process 1 -megapixel gray-scale image, and $120 \mathrm{~ms}$ for color image. The measurement was performed in a PC with an AMD Athlon 64 X2 Dual Core Processor 3800+ 2.00 Ghz and NVIDIA GeForce GT 240. 


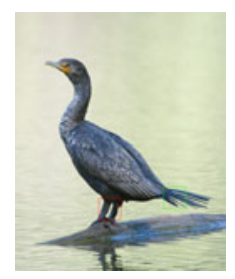

(a) Input

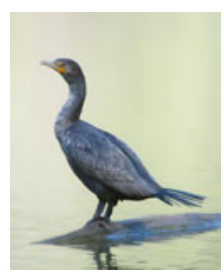

(b) WLS

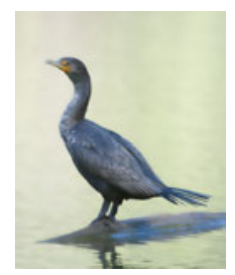

(c) GIF

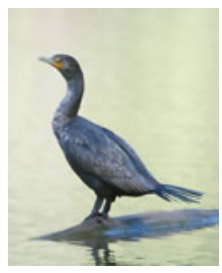

(d) BLF

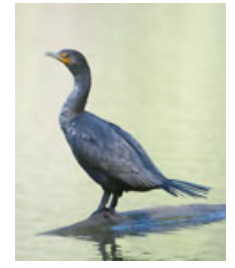

(e) Our

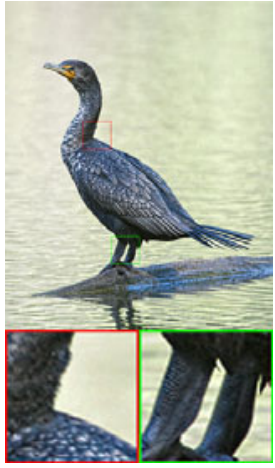

(f) WLS

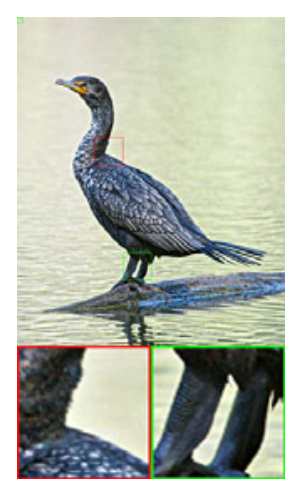

(g) GIF

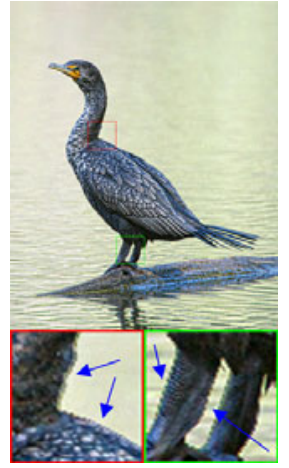

(h) BLF

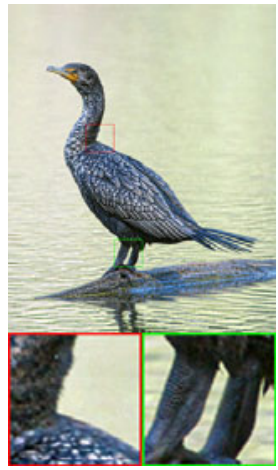

(i) Our

Fig. 4. Filter outputs (top row) and detail increased by $3 \times$ (bottom row) of our method and existing methods for color image. Parameters are configured as follows: WLS: $\lambda=0.2, \alpha=1.2$; GIF: $r=6, \epsilon=0.01$; BLF: $\sigma_{s}=6, \sigma_{r}=0.1$; Our: $\sigma_{s}=6, \sigma_{v r}=0.01$. The input image was taken from [15].

\section{Experimental Results}

\subsection{Detail Enhancement}

Our proposed method focuses on the gradient reversal artifacts occurring in the detail enhancement technique, where the details are first boosted, and then recombined with the base layer to produce an enhanced output. Fig. 4 shows the enhanced results rendered by our method and existing methods. When the artifacts occur near the edges in the BLF result (Fig. 4(h)), our method (Fig. 4(i) greatly reduces these artifacts and can be visually comparable to the enhanced output produced from WLS filter (Fig. 4(f) and GIF (Fig. 4(g)). The same analysis can be applied to the enhanced outputs of gray-scale image as shown in Fig. 5. The reversed gradient artifacts only occur in the BLF result, while the enhanced output produced from our method is comparable to that produced from WLS and GIF.

\subsection{Detail-Preserving Image Denoising}

Denoising is the first and original purpose of image filtering. Especially, smoothing noise, while preserving both edges and fine details in the image simultaneously, is 


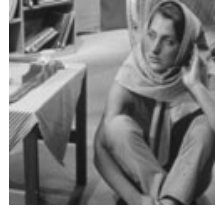

(a) Input

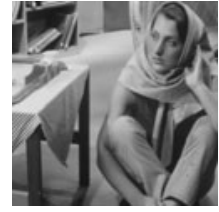

(b) WLS

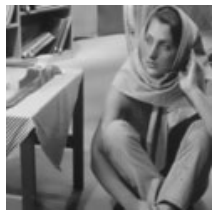

(c) GIF

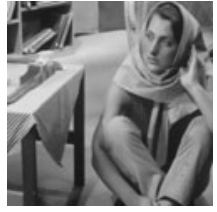

(d) BLF

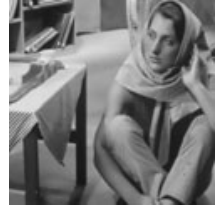

(e) Our

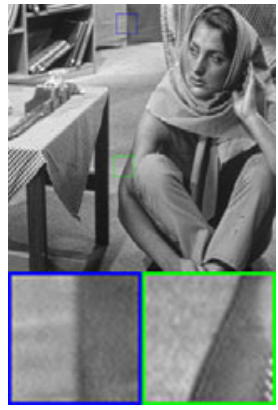

(f) WLS

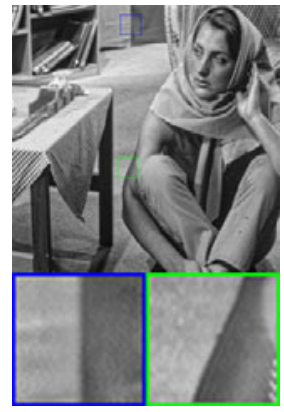

(g) GIF

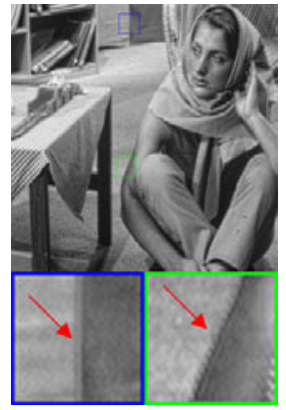

(h) BLF

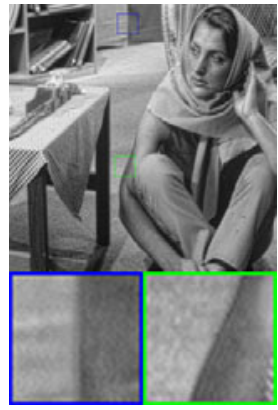

(i) Our

Fig. 5. Filter outputs (top row) and detail increased by $3 \times$ (bottom row) of our method and existing methods for gray-scale image. Parameters are configured as follows: WLS: $\lambda=0.1, \alpha=1.2$; GIF: $r=6, \epsilon=0.05^{2}$; BLF $\sigma_{s}=6, \sigma_{r}=0.05$; Our: $\sigma_{s}=6$, $\sigma_{v r}=0.05^{2}$.

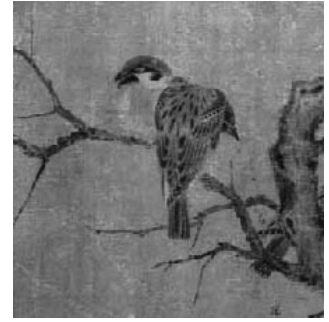

(a) Input image

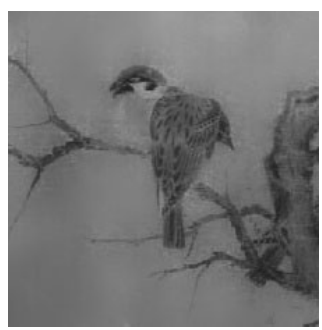

(d) GIF

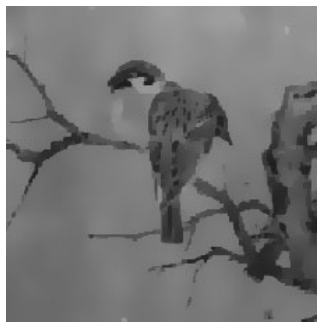

(b) $\mathrm{AD}$

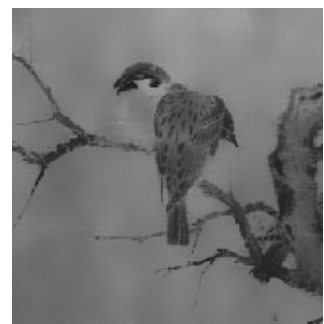

(e) BLF

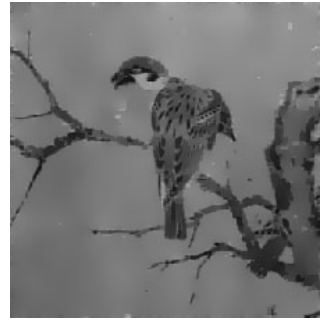

(c) Improved AD

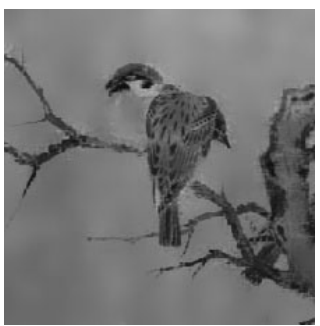

(f) Our

Fig. 6. Comparison of detail-preserving image denoising. Parameters are configured as follows: (b) $\mathrm{AD}: K=3$; (c) Improved AD: $K_{0}=12$; (d) GIF: $r=10, \epsilon=0.01$; (e) BLF: $\sigma_{s}=10, \sigma_{r}=0.1$; (f) Our method: $\sigma_{s}=10, \sigma_{v r}=0.12^{2}$. The input image was taken from [6]. 
an important and challenging task [6]. In terms of restoration using filtering without deploying any noise detection operator, we compare our proposed method with various edge-preserving smoothing techniques including $\mathrm{AD}$ [19], improved AD [6], GIF [14] and BLF [23. The test image is the degraded bird image taken from 6]. While the AD and the improved AD are set to be iterated over 100 times, the smoothing parameters of remaining methods are chosen carefully to achieve tolerable results. The restored image in $\mathrm{AD}$ (Fig. 6(b) has lost most of the details of the bird and the edges are over-sharpened. Though the improved anisotropic diffusion preserves both edges and fine details in the restored image (Fig. 6(c)), it cannot avoid sharpening edges, the same as in anisotropic diffusion. Both GIF and BLF effectively smooth noise in the homogeneous regions, however, the details have been blurred and contrast is reduced, as shown in Fig 6(d) and 6(e), respectively. Our method preserves edges and fine details and avoids sharpening edges, as shown in Fig. 6(f) at the expense of small noise remained near edges. Moreover, the contrast is closer to that of the input image.

\section{Conclusion}

In this paper, we presented an improved BLF model for artifact-free detailand edge-preserving smoothing. The improved model takes into account not only the intensity difference but also the local variance information of the filter windows. It better preserves edges and fine details compared to the standard model. The gradient reversal artifacts have been greatly reduced without using a post-precessing step in detail enhancement, while the meaningful fine details are also preserved in the image denoising technique.

Experimental results showed these detail-related bilateral-based applications can achieve better results by simply switching from the standard model to our proposed model. Its ability to apply to a variety of applications, such as multiscale image decomposition, HDR compression, and flash/no-flash imaging, is limited compared to WLS filter and guided image filter. Further improvement, quantitative evaluations and its relation to non-local filters should be investigated. We leave these challenges for our future works.

\section{References}

1. Aurich, V., Weule, J.: Non-linear gaussian filters performing edge preserving diffusion. In: Proceedings of the DAGM Symposium, pp. 538-545 (1995)

2. Barash, D.: A Fundamental Relationship Between Bilateral Filtering, Adaptive Smoothing, and the Nonlinear Diffusion Equation. IEEE Transactions on Pattern Analysis and Machine Intelligence 24(6), 844-847 (2002)

3. Black, M.J., Sapiro, G., Marimont, D.H., Heeger, D.: Robust anisotropic diffusion. IEEE Transactions on Image Processing 7(3), 421-432 (1998)

4. Buades, A., Coll, B., Morel, J.M.: A non-local algorithm for image denoising. In: IEEE Intl. Conf. on Computer Vision and Pattern Recognition (CVPR), pp. 60-65 (2005) 
5. Buades, A., Coll, B., Morel, J.M.: The staircasing effect in neighborhood filters and its solution. IEEE Transactions on Image Processing 15(6), 1499-1505 (2006)

6. Chao, S.M., Tsai, D.M., Chiu, W.Y., Li, W.C.: Anisotropic diffusion-based detailpreserving smoothing for image restoration. In: IEEE Intl. Conf. on Image Processing (ICIP), pp. 4145-4149 (2010)

7. Chen, J., Paris, S., Durand, F.: Real-time edge-aware image processing with the bilateral grid. ACM Transactions on Graphics 26(3) (2007)

8. Choudhury, P., Tumblin, J.: The trilateral filter for high contrast images and meshes. In: Proceedings of the Eurographics Symposium on Rendering, pp. 186$196(2003)$

9. Dabov, K., Foi, A., Katkovnik, V., Egiazarian, K.: BM3D Image Denoising with Shape-Adaptive Principal Component Analysis. In: Workshop on Signal Processing with Adaptive Sparse Structured Representation (SPARS) (2009)

10. Durand, F., Dorsey, J.: Fast Bilateral Filtering for the Display of High-DynamicRange Images. ACM Transactions on Graphics 21(3), 257-266 (2002)

11. Elad, M.: On the bilateral filter and ways to improve it. IEEE Transactions on Image Processing 11(10), 1141-1151 (2002)

12. Farbman, Z., Fattal, R., Lischinski, D., Szeliski, R.: Edge-Preserving Decompositions for Multi-Scale Tone and Detail Manipulation. ACM Transactions on Graphics 27(3) (2008)

13. Gupta, M.D., Xiao, J.: Bi-affinity Filter: A Bilateral Type Filter for Color Images. In: ECCV Workshop on Color and Reflectance in Computer Vision, CRICV (2010)

14. He, K., Sun, J., Tang, X.: Guided Image Filtering. In: Daniilidis, K., Maragos, P., Paragios, N. (eds.) ECCV 2010, Part I. LNCS, vol. 6311, pp. 1-14. Springer, Heidelberg (2010)

15. Kass, M., Solomon, J.: Smoothed Local Histogram Filters. ACM Transactions on Graphics 29(4) (2010)

16. Levin, A., Lischinski, D., Weiss, Y.: Colorization using Optimization. ACM Transactions on Graphics 23(3), 689-694 (2004)

17. Paris, S., Durand, F.: A Fast Approximation of the Bilateral Filter using a Signal Processing Approach. International Journal of Computer Vision 81(1), 24-52 (2009)

18. Paris, S., Kornprobst, P., Tumblin, J., Durand, F.: Bilateral Filtering: Theory and Applications. In: Foundations and Trends in Computer Graphics and Vision (2009)

19. Penora, P., Malik, J.: Scale-space and edge detection using anisotropic diffusion. IEEE Transactions on Pattern Analysis and Machine Intelligence 12(7), 629-639 (1990)

20. Pham, T.Q., Van Vliet, L.J.: Separable bilateral filtering for fast video preprocessing. In: Proceedings of the IEEE Intl. Conf. on Multimedia and Expo (2005)

21. Smith, S.M., Brady, J.M.: SUSAN - A new approach to low level image processing. International Journal of Computer Vision 23(1), 45-78 (1997)

22. Subr, K., Soler, C., Durand, F.: Edge-preserving Multiscale Image Decomposition based on Local Extrema. ACM Transactions on Graphics 28(5) (2009)

23. Tomasi, C., Manduchi, R.: Bilateral filtering for gray and color images. In: Proceedings of the IEEE Intl. Conf. on Computer Vision (ICCV), pp. 839-846 (1998)

24. Tumblin, J., Turk, G.: LCIS: A boundary hierarchy for detail-preserving contrast reduction. In: ACM SIGGRAPH 1999, pp. 83-90 (1999)

25. Weickert, J.: Anisotropic Diffusion in Image Processing, Stuttgart, Germany (1998)

26. Wong, A.: Adaptive bilateral filtering of image signals using local phase characteristics. Signal Processing 88(6), 1615-1619 (2008)

27. Zhang, B., Allebach, J.P.: Adaptive Bilateral Filter for Sharpness Enhancement and Noise Removal. IEEE Transactions on Image Processing 17(5), 664-678 (2008) 